\title{
Tackling the Minimizers Hiding Behind High-Value Care
}

\author{
Vineet M Arora, MD, MAPP ${ }^{\star *}$, Christopher Moriates, MD²
}

'University of Chicago Pritzker School of Medicine, Director of Educational Initiatives, Costs of Care, Chicago, Illinois; ${ }^{2}$ Department of Medicine, Dell Medical School, The University of Texas, and Director of Implementation Initiatives, Costs of Care, Austin Texas.

W ith the escalating need for academic health centers to control costs, high-value care initiatives targeted at residents have exploded. Recent estimates suggest that more than twothirds of internal medicine residency programs have high-value care curricula. ${ }^{1}$ This growth has been catalyzed, in part, by compelling evidence suggesting that where the residents undergo training is strongly associated with their future utilization. ${ }^{2}$ Although we encourage, support, and participate in high-value care education, as hospitalists, there are potential consequences of the high-value care movement in medical training.

Minimizers - physicians who underestimate the signs and symptoms of a patient, hastily concluding that they have the most benign condition possible - have always existed within residency training. The ethos of "doing nothing" has been around since at least the days of the widely read medical satire House of God. ${ }^{3}$ However, the increasing focus on high-value care creates a socially acceptable banner for minimizers to hide behind when defending inappropriately doing less. For an inpatient with unexplained localized abdominal pain not responding to conservative therapy, a minimizing resident may report to the attending, "They're fine. I am trying to practice high-value care and avoid getting a CT scan."

In their 2011 book, Your Medical Mind, Groopman and Hartzband described how people naturally fall on a scale between medical maximizing and minimizing and how this influences their approach toward healthcare. ${ }^{4}$ Researchers have expanded this construct to create a "Maximizer-Minimizer Scale," which has been used for studying patients and how these traits affect the degree of medical care they receive..$^{5}$ Similar approaches could be used for identifying physicians and trainees at risk of too much minimizer behavior. Although the vast majority of trainees are not minimizers, and overuse continues to be the bigger problem in the majority of academic settings, it is important to understand how the high-value care movement could facilitate minimalist behavior in some residents. Although this article focuses on the educational system, the potential for minimization exists at all levels of clinical prac-

*Corresponding Author: Vineet M. Arora, MD, MAPP, E-mail: varora@uchicago.edu; Telephone: 773-702-8157; Twitter: @FutureDocs

Published online first April 8, 2019.

Received: August 3, 2018; Revised: September 24, 2018; Accepted: October 2, 2018

๑ 2019 Society of Hospital Medicine DOI 10.12788/jhm.3104 tice, including faculty and practicing physicians. Tackling this problem requires understanding the factors that promote the creation of minimizers, how patients and trainees are affected, and the solutions for preventing the spread of minimizers.

\section{FACTORS THAT PROMOTE THE CREATION OF MINIMIZERS}

Several factors may predispose a resident physician to become a minimizer. For example, resident burnout and overwhelming caseloads can contribute to the desire to decrease work by any means necessary. There are several ways a minimizer can accomplish this goal on inpatient rounds. First, a minimizer may present an important or acute problem as an "outpatient issue" that does not require inpatient workup. Second, minimizers may avoid requesting necessary consults, particularly those associated with intensive workups such as neurology, infectious disease, and rheumatology. Minimizers would claim that this is because of a concern of an unnecessary "costly workup," when in reality they fear discovery of new problems, more tests to follow-up, and a potentially prolonged length of stay. Ironically, an institutional focus on hospital throughput can reinforce minimizers since the attending physicians or the hospital administrators may applaud them for avoiding "extra nights" in the hospital.

In addition to high workloads, inadequate clinical expertise favors the creation of minimizers. Although resident physicians may be aware that the probability of a rare disease is low, they may not recognize when ruling it out is appropriate. Thus, they could dismiss subtle cues or patterns that point to the need for further workup. Although attending physicians serve as a safety net, it could take time for them to recognize a resident minimizer who may be presenting biased information that influences their clinical decisions. Moreover, attending physicians may avoid further probing so that they are not perceived as promoting overuse and waste.

\section{DANGERS OF MINIMIZERS}

There are several dangers posed by minimizers, but the most concerning is the impact on patients. Missed diagnoses are a common source of patient maltreatment and contribute to avoidable deaths. ${ }^{6}$ Patients treated by minimizers may continue to experience their acute problem or have to be readmitted because of inadequate treatment. These patients may also lose faith or their trust in the medical system because of inattention to their problems. In fact, minimizing behaviors could have the greatest negative impact on the most vulnerable patients, who often cannot advocate for themselves or who may 
face conscious and unconscious biases, such as assumptions that they are "pain medication-seeking."

In addition to harming patients, minimizers can jeopardize learning opportunities. A minimizer resident squanders the chance to recognize and contribute toward caring for a patient with a rare disease, diminishing their overall clinical development. Other trainees lose the opportunity to learn due to consultations or procedures never obtained. Lastly, as inappropriate attitudes and practices of minimizers spread through the hidden curriculum, particularly to medical students beginning their training, the overall clinical learning environment suffers.

\section{SOLUTIONS FOR PREVENTING THE CREATION OF MINIMIZERS}

There are specific techniques that academic hospitalists and teaching attending physicians can use to help curb the creation of minimizers and promote a clinical learning environment that counters these behaviors. First, instead of focusing on financial costs, it is important for educators to teach the true concept of healthcare value and the primary importance of improving patient outcomes. Embedding appropriateness criteria, such as those from the American College of Radiology, into daily workflows can enable residents to consider not just the cost of imaging but rather the appropriateness given a specific indication. Training programs can provide residents with a closedloop feedback on patient outcomes so that they can recognize whether a diagnosis was missed or a necessary test was not ordered. Additionally, it is critical for residents to understand that improving healthcare value requires taking a big picture view of costs, particularly from the perspective of patients. ${ }^{8} \mathrm{~A}$ patient readmitted after receiving a minimalist workup is more costly to both the patient and the healthcare system.

Second, it is important for the hospitalist faculty to emphasize when a patient has failed a conservative approach and a more specialized, and sometimes intensive, workup or management strategy is appropriate. The classic example is a patient transferred from a community hospital to a tertiary center for further evaluation. Such patients are outside the scope of well-established guidelines. It is precisely these patients that Choosing Wisely ${ }^{\mathrm{TM}}$ or "Less is More" recommendations often do not apply. In contrast, transfer patients often do not end up receiving the specialty procedures that they were originally referred for ${ }^{9}$; it is important that all remain vigilant and committed to high-value care to avoid overuse in these situations.

Exposing residents to cognitive biases is equally important. For example, anchoring can lead to early closure, an easy path for a minimizer to follow. Given the recent focus on the harms related to diagnostic errors, more training in these biases can help promote better patient outcomes. ${ }^{10}$
Lastly, it is critical that hospitalists emphasize the importance of prioritizing a patient's overall health to learners. Although it is tempting for trainees to focus only on acute episodes of a hospital stay, a holistic approach to patients and their quality of life can avoid the minimizer trap. The recent proposal to use home-to-home days in lieu of the routine length of hospital stay is a wonderful example of "measuring what matters to patients" and removing incentives for inappropriately shifting care to other clinicians or venues. ${ }^{11}$ Likewise, alternative payment models for emphasizing patient outcomes over time can create systems that reinforce holistic views of patient health.

\section{CONCLUSION}

The increasing focus on delivering high-value care has created a socially acceptable excuse for minimizers, who could thrive relatively unchecked in the clinical learning environment. To counter this unintended consequence, hospitalists must learn to identify minimizing behavior and actively guard against these tendencies by highlighting the value of appropriate care, not just doing less, and always striving to provide the best care for patients.

Disclosures: Dr. Arora reports personal fees from the American Board of Internal Medicine and personal fees from McGraw Hill, outside the submitted work.

Dr. Moriates reports personal fees from McGraw Hill, outside the submitted work.

\section{References}

1. 2014 APDIM Program Directors Survey- Summary File. http://www.im.org/d/ do/6030. Accessed on July 18, 2017.

2. Chen C, Petterson S, Phillips R, Bazemore A, Mullan F. Spending patterns in region of residency training and subsequent expenditures for care provided by practicing physicians for Medicare beneficiaries. JAMA. 2014;312(22):2385-2393. doi: 10.1001/jama.2014.15973

3. Shem S. The House of God. London, UK: Bodley Head; 1979.

4. Groopman J, Hartzband P. Your Medical Mind: How to Decide What Is Right for You. Reprint edition. New York, NY: Penguin Books; 2012.

5. Scherer LD, Caverly TJ, Burke J, et al. Development of the Medical Maximizer-Minimizer Scale. Health Psychol. 2016;35(11):1276-1287. doi: 10.1037/ hea0000417

6. National Academies of Sciences E. Improving Diagnosis in Health Care.; 2015. https://www.nap.edu/catalog/21794/improving-diagnosis-in-health-care. Accessed September 13, 2018

7. American College of Radiology Appropriateness Criteria. https://www.acr. org/Clinical-Resources/ACR-Appropriateness-Criteria. Accessed on July 28, 2018.

8. Parikh RB, Milstein A, Jain SH. Getting real about health care costs - a broader approach to cost stewardship in medical education. N Engl J Med.2017;376(10):913-915. doi: 10.1056/NEJMp1612517

9. Mueller SK, Zheng J, Orav EJ, Schnipper JL. Interhospital transfer and receipt of specialty procedures. J Hosp Med. 2018;13(6):383-387. doi: 10.12788/ jhm.2875

10. Trowbridge RL, Dhaliwal G, Cosby KS. Educational agenda for diagnostic error reduction. BMJ Qual Saf. 2013;22(2 Suppl):ii28-ii32.

11. Barnett ML, Grabowski DC, Mehrotra A. Home-to-home time - measuring what matters to patients and payers. N Engl J Med. 2017;377(1):4-6. 